\title{
A catalog of edge-on disk galaxies ${ }^{\star}$
}

\section{From galaxies with a bulge to superthin galaxies}

\author{
S. J. Kautsch ${ }^{1}$, E. K. Grebel ${ }^{1}$, F. D. Barazza ${ }^{1,2}$, and J. S. Gallagher $\mathrm{III}^{3}$
}

\author{
1 Astronomical Institute, Department of Physics and Astronomy, University of Basel, Venusstrasse 7, 4102 Binningen, Switzerland \\ e-mail: kautsch@astro.unibas.ch \\ 2 Department of Astronomy, University of Texas at Austin, 1 University Station C1400, Austin, TX 78712-0259, USA \\ 3 Department of Astronomy, University of Wisconsin, 475 North Charter Street, Madison, WI 53706-1582, USA
}

Received 3 August 2005 / Accepted 2 September 2005

ABSTRACT

Spiral galaxies range from bulge-dominated early-type galaxies to late types with little or no bulge. Cosmological models do not predict the formation of disk-dominated, essentially bulgeless galaxies, yet these objects exist. A particularly striking and poorly understood example of bulgeless galaxies are flat or superthin galaxies with large axis ratios. We therefore embarked on a study aimed at a better understanding of these enigmatic objects, starting by compiling a statistically meaningful sample with well-defined properties. The disk axis ratios can be most easily measured when galaxies are seen edge-on. We used data from the Sloan Digital Sky Survey (SDSS) in order to identify edge-on galaxies with disks in a uniform, reproducible, automated fashion. In the five-color photometric database of the SDSS Data Release $1\left(2099\right.$ deg$\left.^{2}\right)$ we identified 3169 edge-on disk galaxies, which we subdivided into disk galaxies with bulge, intermediate types, and simple disk galaxies without any obvious bulge component. We subdivided these types further into subclasses: $\operatorname{Sa}(f), \operatorname{Sb}(f), \operatorname{Sc}(f), \operatorname{Scd}(f), \operatorname{Sd}(f), \operatorname{Irr}(f)$, where the (f) indicates that these galaxies are seen edge-on. Here we present our selection algorithm and the resulting catalogs of the 3169 edge-on disk galaxies including the photometric, morphological, and structural parameters of our targets. A number of incompleteness effects affect our catalog, but it contains almost a factor of four more bulgeless galaxies with prominent simple disks (flat galaxies) within the area covered here than previous optical catalogs, which were based on the visual selection from photographic plates (cf. Karachentsev et al. 1999, Bull. Special Astrophys. Obs., 47, 5). We find that approximately $15 \%$ of the edge-on disk galaxies in our catalog are flat galaxies, demonstrating that these galaxies are fairly common, especially among intermediate-mass star-forming galaxies. Bulgeless disks account for roughly one third of our galaxies when also puffy disks and edge-on irregulars are included. Our catalog provides a uniform database for a multitude of follow-up studies of bulgeless galaxies in order to constrain their intrinsic and environmental properties and their evolutionary status.

Key words. catalogs - galaxies: spiral - galaxies: irregular - galaxies: photometry - galaxies: statistics - galaxies: fundamental parameters

\section{Introduction}

During the last decade an increasing number of studies of latetype edge-on disk-dominated galaxies has been conducted, reflecting a growing interest in understanding these galaxies in the framework of galaxy evolution and cosmological models. Models describing the chemodynamical evolution of disk galaxies within a slowly growing dark matter halo can successfully reproduce many of the observed properties of MilkyWay-type disk galaxies (Samland \& Gerhard 2003; Samland 2004). Models with high merger rates as mandated in hierarchical merger scenarios face a number of problems when comparing the predicted properties of galactic subcomponents with observations (e.g., Abadi et al. 2003). It is even more

* The full catalog tables (Tables 2 and 3) are only available in electronic form at the CDS via anonymous ftp to cdsarc.u-strasbg. fr $(130.79 .128 .5)$ or via

http://cdsweb.u-strasbg.fr/cgi-bin/qcat?J/A+A/445/765 difficult to succeed in producing disk-dominated, essentially bulge-less late-type galaxies, making these objects an evolutionary enigma. In cold dark matter (CDM) simulations the resulting disks are smaller, denser, and have lower angular momentum than observed. Major mergers increase the angular momentum (e.g., Gardner 2001), but also destroy disks, hence it seems unlikely that simple disk galaxies suffered major mergers in the recent past. Adding feedback alleviates the angular momentum problem to some extent (e.g., Sommer-Larsen et al. 2003; Robertson et al. 2005). However, D’Onghia \& Burkert (2004) point out that dark halos that did not suffer major mergers have too low an angular momentum to begin with. This prevents them from producing the observed extended disks from the collapse of their associated baryons, since the specific angular momentum of the gas cannot be increased by feedback processes.

Overall, disk galaxies show a multitude of different morphologies ranging from disk galaxies with a substantial 
bulge and with high surface brightness to bulgeless lowsurface-brightness (LSB) galaxies (e.g., Schombert et al. 1992; Matthews et al. 1999) and various complex bulge/disk combinations in between (e.g., Matthews \& de Grijs 2004). While certain properties such as the asymptotically flat rotation curves seem to be shared by most disk galaxies, they differ in other key properties such as surface brightness and scale length. In order to understand how these systems form and evolve we need to understand the morphological systematics from bulgeless disks to disk galaxies with a dominant spheroidal bulge.

Historically, models consider disk formation as the result of the collapse of a gaseous protogalaxy (e.g., Fall \& Efstathiou 1980). Disks may then form from inside-out around a preexisting classical bulge (Athanassoula 2005) or via smooth accretion of material (Steinmetz \& Navarro 2002). On the other hand, intense star formation will also lead to the formation of a bulge or a dense nucleus in a bulgeless disk galaxy, aided by the rapidity of the gas infall and the total amount of the accreted material (either through infall or mergers) (Noguchi 2001). Also bars, formed via instabilities of a disk, can transport material to the disk center. The subsequent star formation may build up an additional bulge component, which can then stabilize the disk (Samland \& Gerhard 2003).

Dalcanton et al. (1997) propose a scenario where gas in low angular momentum protogalaxies collapses efficiently, resulting in high-surface-brightness galaxies. Protogalaxies with high angular momentum and lower mass, on the other hand, evolve into LSB galaxies. Dalcanton et al. (1997) note that "gravitational collapse in any hierarchical model with Gaussian initial conditions leads to a broad distribution of halo masses and angular momenta", which could account for the observed range of properties. Dalcanton et al. (2004) found that galaxies with disk circular velocities $V_{\mathrm{c}}>120 \mathrm{~km} \mathrm{~s}^{-1}$ tend to show bulges. They suggest that these objects are more gravitationally unstable, which can lead to fragmentation and gravitational collapse along spiral arms and subsequently to smaller gas scale heights, pronounced dust lanes, and star formation. In this picture, slowly rotating disks are stable and have low star formation rates, implying also lower metallicities.

While these scenarios offer a convincing and internally consistent explanation for the nature of disk galaxies, the frequency and stability of disk-dominated galaxies is surprising from the cosmological point of view. Hierarchical models of galaxy formation include violent interaction phases that should destroy disky systems (Steinmetz 2003; Taylor \& Babul 2003). A better knowledge of disk-dominated galaxies may hence be key for understanding their formation, evolution, and survival.

The need for a homogeneous search for mainly bulgeless edge-on galaxies was recognized by Karachentsev (1989), who wanted to use these objects in order to investigate large-scale streaming motions in the universe. He used photographic data in order to identify and catalog these systems. The resulting catalogs are the "Flat Galaxy Catalogue" (hereafter FGC) (Karachentsev et al. 1993) and its extension, the "Revised Flat Galaxy Catalogue" (hereafter RFGC) by Karachentsev et al. (1999). This RFGC is an all-sky survey and contains the largest published compilation of visually selected bulgeless edge-on galaxies: 4236 objects in total. A collection of edge-on disk galaxies in the near infrared is provided in "The 2MASSselected Flat Galaxy Catalog" (Mitronova et al. 2004). Since the appearance of these highly inclined disks is essentially needle-like and does not exhibit a distinct bulge component Karachentsev (1989) called them "flat galaxies". Flat galaxies are thin edge-on spiral galaxies which seem to be (nearly) bulgeless and of late morphological Hubble type ( $\mathrm{Sc} / \mathrm{Sd}$ and later). A few years earlier, Goad \& Roberts (1979) and Goad \& Roberts (1981) already called attention to edge-on galaxies with extreme axial ratios. They called these systems "superthin galaxies". Superthin and flat galaxies belong to the same group, which we will summarize here under the term "simple disk galaxies".

In order to contribute to a better characterization of these objects, we carried out the work presented here, which aims at compiling a uniform sample of disk-dominated galaxies from modern CCD data at optical wavelengths. The Sloan Digital Sky Survey (SDSS) with its homogeneous, deep, large-area coverage provides an ideal data base for the identification of such galaxies. The SDSS (York et al. 2000) is carrying out multi-color imaging of one quarter of the sky, followed by medium-resolution spectroscopy primarily of galaxies and other objects of interest down to certain magnitude limits. These data are pipeline-reduced and the resulting images, astrometry, photometry, structural parameters, and calibrated spectra are released to the public after a proprietary period (Stoughton et al. 2002; Abazajian et al. 2003, 2004, 2005).

The SDSS with its resolution, dynamic range, and photometric accuracy allows one to study statistical properties and biases of disk galaxies such as their structure, intrinsic properties, overall frequency, and global scaling relations. The formation and evolution scenarios can be probed by studying the detailed structure and morphology (e.g., bulges, bars, halos, knots, and lanes) and comparing these with predictions from models (e.g., Samland \& Gerhard 2003; Samland 2004; Immeli et al. 2004). Also the frequency of warps of the edge-on galaxies and possible relations with the environment can be studied easily using the SDSS. Warps should be relativy frequent since Reshetnikov (1995) showed that about $40 \%$ of the FGC galaxies have pronounced warps. Radial and vertical color gradients in these systems can shed light on the assembly of structure and on the evolutionary state using the available multi-color photometry.

In addition, the SDSS spectra enable the estimation of the properties of the stellar populations, of the star formation rates, central activity, and metallicities. The redshifts from the catalog allow one to estimate the luminosities and sizes of the galaxies and the distribution of these properties. Also the environment of the cataloged galaxies can be investigated to probe the distribution of the surrounding satellites, the Holmberg effect, external influences on morphological evolution, and the local density and properties (frequency, position, and alignment) in a cluster environment.

We confined our search of the SDSS data base to edge-on disk galaxies, which facilitates the definition of an effective selection criterion. The choice of edge-on galaxies in particular is the only way to reliably select pure disk galaxies based on their optical morphologies. Altogether, we collected 3169 edge-on 
galaxies from the SDSS Data Release 1 (SDSS DR1). These systems can be subdivided into subclasses according their appearance.

This paper is organized as follows: in Sect. 2 we describe the training set of galaxies and the resulting selection criteria. In Sect. 3 the actual target selection is explained. The classification of the detected objects is presented in Sect. 4, followed by a description of the catalog (Sect. 5). Comparisons to evaluate the completeness of our selection are presented in Sect. 6. The influence of dust extinction on the galaxy selection is discussed in Sect. 7. Different types of galaxies and their subclasses are discussed in Sect. 8. The last Sect. 9 contains the summary and conclusions.

\section{Training set and selection criteria}

\subsection{The data base}

Our intent is to find edge-on galaxies with dominant stellar disks. We are using Karachentsev et al.'s catalogs as a starting point in order to carry out a systematic and reproducible selection of these kinds of galaxies. The object selection in the FGC and RFGC was based on the visual identification of galaxies with an axial ratio of $a / b \geqslant 7$ and a major axis diameter $a \gtrsim 40^{\prime \prime}$ in the blue band on copies of the POSS-I and ESO/SERC photographic plates. The availability of the SDSS database permits us to carry out a survey using deep, homogeneous, five-color CCD data that are superior to the less deep, inhomogeneous photographic plates. An added advantage of the SDSS is that it will ultimately allow us to carry out such a search in an automated, objective, repeatable fashion. This certainly does not render the earlier studies superfluous since the SDSS is not an all-sky survey and since the earlier identifications provide a valuable training set for the definition of the selection criteria to be applied to the digital data. Furthermore, the SDSS permits us to identify not only simple-disk candidates, but also edge-on galaxies in general and to investigate the properties of all of these different morphological types.

We have analyzed SDSS data from DR1 (Abazajian et al. 2003), which was the largest publicly available data set when this work was started. DR1 provides $2099 \mathrm{deg}^{2}$ of imaging data observed in the five SDSS filters ugriz. The $r$-band depth of these data is approximately $22.6 \mathrm{mag}$. Meanwhile the data releases 2 (DR2) (Abazajian et al. 2004), 3 (DR3) (Abazajian et al. 2005), and 4 (DR4) (Adelman-McCarthy et al. 2006) are available, which cover successively larger areas on the sky. As detailed in Abazajian et al. (2004), changes were made to the data processing software between DR1 and DR2, but no such changes occurred for DR3 as compared to DR2 (Abazajian et al. 2005). We compared the SDSS photometry parameters in DR1 and DR2 for our galaxies and found no significant changes. However, in all of the releases some galaxies are affected by so-called "shredding" (e.g., Abazajian et al. 2003, 2004; Kniazev et al. 2004a), i.e., these galaxies are detected as two or more independent objects. This is found in particular for extended objects with substructure and diameters $\geqslant 1^{\prime}$. A comparison of the different data releases showed that shredded target galaxies are similarly miss-classified in all of these releases. Some of the galaxies that were correctly identified in DR1 turned out to be shredded in the later releases. Hence we decided to continue to work with DR1 for the pilot study presented here.

\subsection{Definition of a training set}

In order to quantify a training set for the selection of diskdominated edge-on galaxies we searched for all RFGC galaxies with a right ascension between 000000 and 021200 in the DR1 database, using the RFGC coordinates. In this coordinate range we expect to recover 47 RFGC galaxies in the DR1. It turned out that two of these objects have significantly different coordinates from the galaxies detected in the SDSS, while a third galaxy has a very different angular diameter in the RFGC as compared to the SDSS. For the remainder, the difference between the RFGC coordinates and the SDSS coordinates is typically smaller than \pm 0.001 degrees $\left(3.6^{\prime \prime}\right)$. For this "training set" that we re-identified in the SDSS, we found that the structural parameters have slightly smaller values in the DR1 as compared to the RFGC. We tested various combinations of SDSS structural and photometric parameters that would allow us to recover the galaxies in the training set (and additionally other edge-on disk galaxies in the SDSS). Ultimately, these galaxies should be recovered by performing an automated search of the SDSS photometric catalog database.

\subsection{Definition of the query}

As the result of this empirical approach, we finally adopted the parameters listed below for subsequent queries of the DR1 "Best Galaxy Table" (Abazajian et al. 2003). The DR1 "Best Galaxy Table" is the table in the SDSS database containing all parameters for galaxies that are of the highest quality at the time of the data release.

- Axial ratio in the g band: $a / b>3$, where $a$ and $b$ are the major and minor axis, respectively.

- Angular diameter (isophotal major axis of the galaxy in the "blue" g filter) $a>30 "$ ".

- Colors in the range of $0.5<g-r<2$ mag and $0.5<r-i<$ 2 mag.

- Magnitude limit in the $\mathrm{g}$ filter $<20 \mathrm{mag}$.

With these conditions we are able to essentially reproduce all of the RFGC criteria and to recover the training set. In addition to the RFGC galaxies, our query parameters yield a much larger number of flat edge-on galaxies and other disk-dominated objects. This increase in numbers is in part due to the higher resolution and depth of the DR1 as compared to the photographic plates, but also due to our intention to collect all edge-on disk galaxies (including those with bulges). The latter is facilitated particularly by our relaxed choice of minimum axis ratios.

The images of the thus found objects were then downloaded from the DR1 "Data Archive Server" (DAS) using the SDSS rsync server. We downloaded the so-called "corrected imaging frames" (fpC) in the five SDSS bands. For a detailed 
description of the $\mathrm{fpC}$ frames we refer to the on-line description in the SDSS webpages ${ }^{1}$.

\section{Target selection}

After a visual inspection we removed contaminants from our object list. The contaminants are mostly spikes (from very bright stars) and artifacts such as satellite or meteorite tracks which resemble an edge-on galaxy. Also strongly spikeblended edge-on galaxies were rejected. Additionally, obvious non-edge-on systems and unknown objects were removed. The obvious non-edge-on systems are objects where a bright bar in a faint disk simulates an edge-on disk. Apart from these contaminants, our selection criteria produce a fairly uniform sample of extended disk-dominated galaxies including objects with bulges and bulgeless simple disks. All in all, 3169 objects were assembled in our catalog. Some early-type edge-on spiral galaxies are also included in the catalog, but internal dust lanes introduce a bias in excluding a fraction of these galaxies. This will be discussed in greater detail in Sect. 7. Additionally, our sample is limited by our selection criteria and by the SDSS photometry itself. The following biases affect our selection: (1) Edge-on galaxies with very faint disks around bulges and bright centers. (2) "Shredded galaxies". (3) Galaxies with unusual colors caused by an AGN and/or dust.

The edge-on galaxies remaining in our sample after visual inspection and removal of contaminants fall into three general morphological groups:

- pure bulgeless disks/simple disks;

- galaxies with a disk and an apparent bulge;

- objects with disks and central light concentration but no obvious bulge-like structure. These may be considered an intermediate class between the simple disks and galaxies with bulges. This group also comprises edge-on disky irregulars.

Out of these galaxies, an effort was made to select by eye objects spanning the full range in properties including different disk thicknesses, different bulge sizes, and presence or absence of dust lanes. The result is a subsample of 129 galaxies that is our morphological "reference set". Via visual inspection we subdivided this reference set into 42 simple disks, 37 galaxies with a bulge, and 50 intermediate types with central light excess. We then used this subsample to further automate the separation process and to develop a code to recover these general classes of edge-on galaxies in the SDSS DR1.

We found that the luminosity-weighted mean value of the ellipticity (hereafter $\varepsilon$ ) of the elliptical isophotes is a very robust separator between simple disks and the other edge-on types. In combination with the concentration index (hereafter CI) we can also exclude galaxies with an apparent bulge. The CI clearly separates galaxies with bulge from those without an apparent spheroidal component. This will be detailed in the following sections.

\footnotetext{
${ }^{1}$ http://www.sdss.org/dr1/dm/flatFiles/fpC.html
}

\subsection{Isophote fitting}

The following analysis is performed with the MIDAS analysis package developed by the European Southern Observatory. We applied it to the frames in all five SDSS filters, but only used the results for the three most sensitive bands gri. Unless explicitly specified otherwise, magnitudes quoted below refer to each of the separate bands. Firstly, we subtract the sky and the "softbias" from all frames. The softbias is an additional offset of 1000 counts per pixel in order to avoid negative pixels in the images. The sky and softbias were subtracted as mean values from the images. The values of the sky and softbias are stored in the header of each $\mathrm{fpC}$ frame. Then we use the MIDAS surface photometry package "surfphot" to fit ellipses to the isophotes of our galaxies. The innermost ellipse is fitted adopting the center coordinates given by the DR1 photometric database. The intensity of the innermost isophote is derived from the luminosity of the brightest pixel in a box $(8 \times 8$ pixel $)$ that corresponds to the galaxy center.

In steps of 0.2 mag the program fits ellipses until an isophote is reached that corresponds to a surface brightness of $\mu=25 \mathrm{mag} \operatorname{arcsec}^{-2}$. This implies that on average 20-30 isophote levels are plotted for every galaxy depending on the size and brightness. This isophote algorithm is based on the formulae of Bender \& Moellenhof (1987).

\subsection{Measuring the luminosity-weighted mean ellipticity and concentration index}

We use the resulting values of the isophote levels and the major (a) and minor (b) axes in order to derive the luminosity weighted mean ellipticity of the elliptical isophotes $(\varepsilon) . \varepsilon$ is defined as

$\varepsilon=\frac{\sum_{i=1}^{n} \epsilon_{i} \cdot I_{i}}{\sum_{i=1}^{n} I_{i}}$

and

$\epsilon_{i}=1-\frac{b_{i}}{a_{i}}$

is the ellipticity of the $i$ th isophote, whereas

$I_{i}=z_{i} \cdot\left((a \cdot b)_{i}-(a \cdot b)_{i-1}\right) \cdot \pi$

is the intensity between two isophote levels. The isophote level is indicated by $z_{i}$.

For the CI of these objects we used the ratio of the following SDSS parameters

$C I=$ petrorad $\_90 \cdot$ petrorad $\_50^{-1}$.

This is the ratio of the Petrosian radii (petrorad) that contain $90 \%$ and $50 \%$ of the Petrosian flux in the same band, respectively (see Stoughton et al. 2002). The Petrosian radius is the radius of a circular aperture at which the "Petrosian ratio" is set to a fixed value of 0.2 . This "Petrosian ratio" is the ratio of the surface brightness in an annulus at a certain radius to the mean surface brightness within a circle with this radius. As discussed 


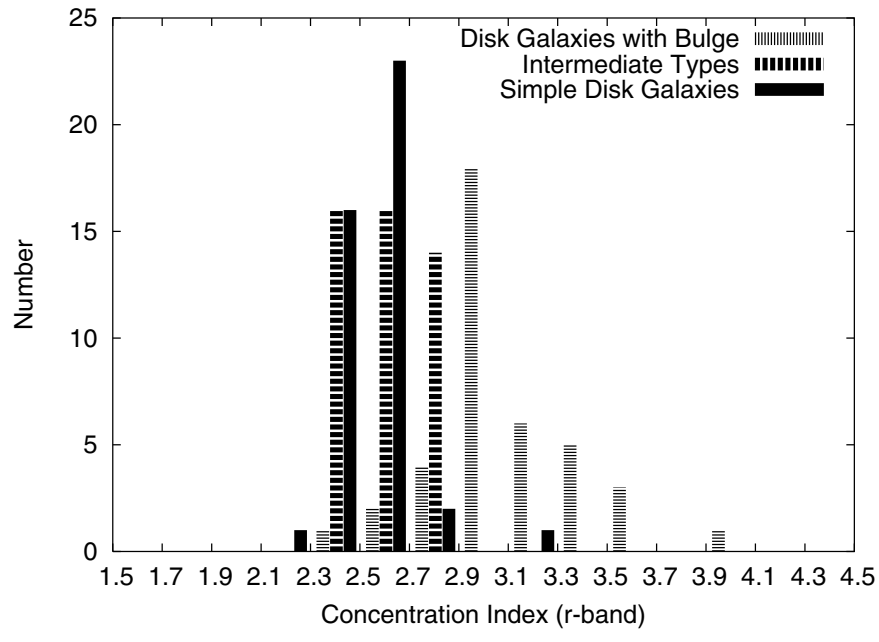

Fig. 1. Number distribution of the visually selected galaxies versus their concentration index (CI).

in Strauss et al. (2002) the use of circular apertures instead of elliptical apertures is fairly insensitive to inclination. Similarly, the Petrosian magnitudes are derived from the Petrosian flux using a circular aperture centered on every object. The advantage of this method is that this allows an unbiased measurement of a constant fraction of the total galaxy light using the technique based on that of Petrosian (1976). For a detailed description of the Petrosian parameters used in the SDSS we refer to Blanton et al. (2001) and Yasuda et al. (2001).

The CI is known as a morphological separator between early- and late-type galaxies (see, e.g., Strateva et al. 2001; Shimasaku et al. 2001; Nakamura et al. 2003; Shen et al. 2003; Kniazev et al. 2004a). With the CI and with $\varepsilon$ as separators we recover our visually selected subgroups of the training set very well automatically. Therefore we applied this procedure to all edge-on galaxies in our catalog.

\subsection{Choosing the limiting values of $\mathrm{Cl}$ and $\varepsilon$}

In order to determine which choices of CI distinguish best between the general types of edge-on galaxies we use a histogram with the distribution of the CIs of the visually "classified" galaxies in our reference set (Fig. 1). Clearly, the majority of the simple disk galaxies has a $\mathrm{CI}<2.7$, hence we adopt a CI of 2.7 as the boundary condition to differentiate between simple disks and intermediate-type galaxies from those of with an obvious bulge component. A slightly lower value was often used in previous morphological studies of galaxies from the SDSS in order to separate between S0/Sa-type spirals and later spiral types (Strateva et al. 2001; Shimasaku et al. 2001; Nakamura et al. 2003; Shen et al. 2003). Contrary to our study these authors did not limit their samples to edge-on galaxies. Disky irregular galaxies exhibit very low CIs: the limit is CI $<2.15$ (see also discussion in Kniazev et al. 2004a). Unfortunately it is not possible to separate the intermediate type from simple disks using only the CI. As one can see in the histogram the two remaining classes are merged at low values of the CI despite their different morphological appearance.

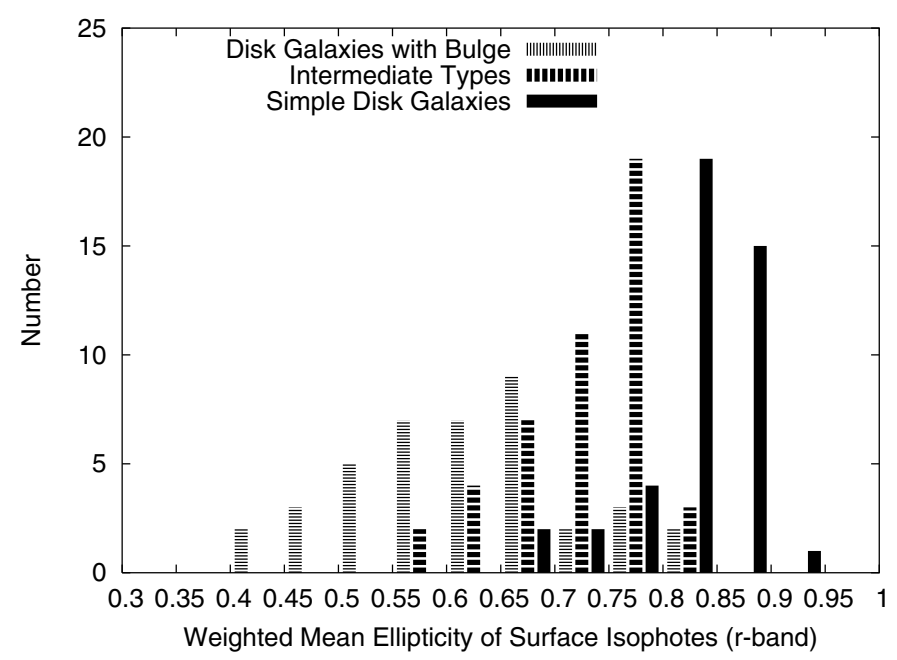

Fig. 2. Number distribution of the visually selected galaxies versus their weighted mean ellipticity of the isophotes $(\varepsilon)$.

For that reason we use the weighted mean ellipticity of the isophotes $\varepsilon$ as the second discriminator. Other possible morphological separators from the literature such as colors, asymmetry index, and profile likelihoods (Strateva et al. 2001), as well as decomposition (Kelly \& McKay 2004) and Gini index (Abraham et al. 2003; Lotz et al. 2004) turned out not to be useful for the sensitive characterization of edge-on galaxies (Kautsch \& Grebel 2003) probably because of the influence of dust and of the galaxy inclination on these separators.

We again use a histogram of the number distribution (Fig. 2) of the $\varepsilon$ values of the training subsample. In this case we intend to separate the intermediate types from the simple disks. We defined the region of $0.75 \leq \varepsilon<0.8$ as the transition zone between the classes and a value of $\varepsilon=0.8$ as the sharp border. This limiting value allows us to select the best simple disk candidates and the transition types. Additionally, with $\varepsilon<0.55$ we can divide the class of galaxies with a bulge into early and later types.

\section{The classification of edge-on galaxies}

In order to flag these systems we follow the terminology introduced by de Vaucouleurs (1959). In his scheme, spiral galaxies are marked with an additional letter referring to the shape of the spiral arms, e.g., "r" means ring shaped and "s" s-shaped spiral structure when seen face on. We will instead use an " $f$ " to indicate that a galaxy is flat, i.e., contains an edge-on component with or without a bulge. Furthermore, we introduce the following subclasses: galaxies with bulges ( $\mathrm{Sa}(\mathrm{f}), \mathrm{Sb}(\mathrm{f}))$; simple disks $(\operatorname{Sd}(\mathrm{f})), \operatorname{Sc}(\mathrm{f})$ and an intermediate group between $\operatorname{Sc}(\mathrm{f})$ and $\operatorname{Sd}(\mathrm{f})$ called $\mathrm{Scd}(\mathrm{f})$, and disky edge-on irregulars (Irr(f)). Representative examples of the general class members are shown in Fig. 3. The three galaxies shown are for the Sb(f) class (SDSS J020405.91-080730.3), for the Scd(f) class (SDSS J102903.90+611525.8), and for the Sd(f) class (SDSS $\mathrm{J} 135309.65+045739.3)$. These galaxy images are three-color $(g, r, i)$ composites provided by the SDSS DR3 Image List Tool and have a scale of $90^{\prime \prime}$ in $\mathrm{X}$ and $\mathrm{Y}$ direction. The separation diagram, Fig. 4, exhibits CI and $\varepsilon$ in order to separate these 

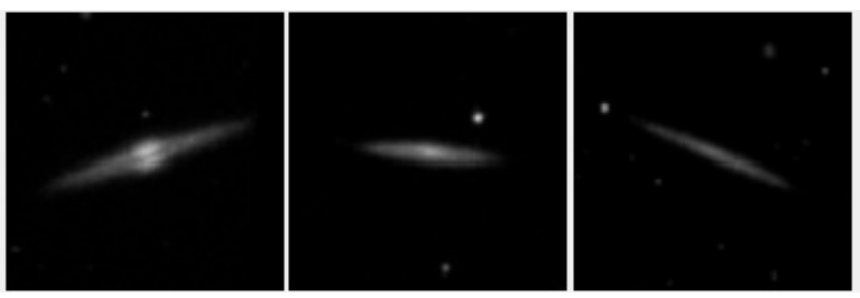

Fig. 3. The left image is a typical member of the class of galaxies with bulge Sb(f): SDSS J020405.91-080730.3. A typical example of the $\operatorname{Scd}(\mathrm{f})$ intermediate class is in the middle: SDSS J102903.90+611525.8. Simple disk galaxies Sd(f) have an appearance like SDSS J135309.65+045739 at the right. All images are cutouts from the DR3 Image List Tool. These images have a scale of 90 square arcsec.

classes. The abscissa represents the luminosity-weighted mean ellipticity of the isophotes $\varepsilon$. The ordinate shows the concentration index CI as taken from the SDSS. The values are given in the SDSS $r$ band ${ }^{2}$. The automatically recovered simple disk galaxies are hereafter marked with $\mathrm{Sd}(\mathrm{f})$, the intermediate types with $\operatorname{Scd}(\mathrm{f})$ and $\mathrm{Sc}(\mathrm{f})$, the galaxies with bulges with $\mathrm{Sa}(\mathrm{f})$ and $\mathrm{Sb}(\mathrm{f})$, and irregulars with $\operatorname{Irr}(\mathrm{f})$. The lines mark the borders between the general classes and the subtypes in our new classification within the general classes. The selection parameters for the $g, r, i$ bands are listed in Table 1 .

In the histogram in Fig. 5 we plotted the number distribution of the apparent diameters of our galaxies. The majority of our objects is smaller than $60^{\prime \prime}$ ( $\sim 88 \%$ of all catalog objects). Only $\sim 2 \%$ of all galaxies in our catalog have a diameter larger than 100" (Fig. 5). In order to check the influence of the size of the objects on our separation we plotted galaxy samples with different angular size (less than and greater than 60") in Fig. 6. This permits us to test whether higher resolution affects the separation process, in particular whether bright centers and extended disks bias the classification. The upper inset shows the number distribution of the CIs. Both size samples follow the same distribution. In the bottom inset the number distribution of $\varepsilon$ is presented.

Galaxies with diameters $a>60^{\prime \prime}$ tend to have slightly higher values of $\varepsilon$, since these galaxies tend to be closer to us, facilitating the detection of more highly eccentric isophotes in the outer regions of these extended objects.

Using visual inspection we found that all $\mathrm{Sd}(\mathrm{f})$ types with an angular diameter $a>60^{\prime \prime}$ show the appearance of a simple disk. Consequently they are assigned to the correct class by the automated algorithm. We therefore conclude that the defined limiting values of our catalog are still robust enough so that size

\footnotetext{
${ }^{2}$ For the selection from the photometric database we used the $g$ band in order to make the parameters comparable to those used for selecting the FGC. In the following diagrams, however, we refer to the $\mathrm{r}$ band. This filter is mostly used in the other studies involving the CI (Shimasaku et al. 2001; Nakamura et al. 2003; Shen et al. 2003) because its quantum efficiency is the highest of all SDSS bands (Stoughton et al. 2002). In addition it includes the red light of the bulge which is important to separate galaxies with bulges from bulgeless galaxies.
}

Table 1. Limiting values. These are the values of the limits of the morphological classes. The values are valid for the SDSS $g$ and $r$ bands. The value for the $i$ band is the same as in the other filters for $\varepsilon$. For $\mathrm{CI}$ it is slightly higher because $i$ is more sensitive for the dominant redder bulge stars. In this case one should add a value of 0.1 to the numbers of the $\mathrm{CI}$ in this table. In general, note that the galaxies near boundaries have the least certain classification.

\begin{tabular}{rrr|rr}
\hline \hline Class & \multicolumn{2}{c}{$\varepsilon$} & \multicolumn{2}{c}{ CI } \\
\cline { 2 - 5 } & lower limit & upper limit & lower limit & upper limit \\
\hline $\mathrm{Sa}(\mathrm{f})$ & - & $<0.55$ & $\geq 2.70$ & - \\
$\mathrm{Sb}$ (f) & $\geq 0.55$ & - & $\geq 2.70$ & - \\
$\mathrm{Sc}(\mathrm{f})$ & - & $<0.75$ & $\geq 2.15$ & $<2.70$ \\
$\mathrm{Scd}(\mathrm{f})$ & $\geq 0.75$ & $<0.80$ & $\geq 2.15$ & $<2.70$ \\
$\mathrm{Sd}(\mathrm{f})$ & $\geq 0.80$ & - & - & $<2.70$ \\
$\operatorname{Irr}(\mathrm{f})$ & - & $<0.80$ & - & $<2.15$ \\
\hline
\end{tabular}

and resolution do not affect the classification. The influence of resolution on the separation is discussed in Sect. 7.

\section{The catalog}

The main catalog is listed in Tables 2 and 3. The structural parameters of the catalog entries are shown in Table 2. Table 3 contains the photometric parameters and the redshifts. All entries are ordered by increasing right ascension. The full tables are available from the CDS. These tables contain all edge-on galaxies with disks that fulfill our automatic selection criteria, ranging from early-type spirals to late-type spirals and irregulars. In total, our catalog contains 3169 objects.

Table 2 is organized as follows: Col. (1) presents the galaxy name in the SDSS nomenclature, which is consistent with the IAU nomenclature requirements. The following two Cols. (2) and (3) contain the coordinates of the galaxies, i.e., right ascension and declination for the epoch J2000. Column (4) indicates the general class (simple disks: $\mathrm{Sd}(\mathrm{f})$; intermediate types: $\operatorname{Sc}(f), \operatorname{Scd}(f)$ and $\operatorname{Irr}(f)$; galaxies with bulge: $\mathrm{Sa}(\mathrm{f})$ and $\mathrm{Sb}(\mathrm{f})$ ). The Cols. (5)-(7) show our derived value of $\varepsilon$ in the $g, r$, and $i$ bands. Columns (8)-(10) contain the CI in these same bands. The angular diameter in $g, r$, and $i$ in arcsec is presented in Cols. (11)-(13). The axial ratios in the $g, r, i$ bands are derived from the ratio of iso $A /$ iso $B$ of the SDSS parameters and are listed in Cols. (14)-(16). iso $A$ is the isophotal major axis and iso $B$ the isophotal minor axis of an isophote with a surface brightness of $\mu=25 \mathrm{mag} \operatorname{arcsec}^{-2}$ (in the respective band) as given by the DR1 pipeline.

Table 3 starts with the SDSS designation of the galaxy (Col. (1)) followed by our proposed class in Col. (2). Petrosian magnitudes and their uncertainties are provided in the $g, r$, and $i$ bands in Cols. (3) to (8). The total surface brightness in $g, r, i$ is given in Cols (9), (10), and (11). We derived it using the parameters petroMag + rho, which are given in the SDSS DR1 database. In this database, rho is five times the logarithm of the Petrosian radius in the $i$ band. The Petrosian magnitudes and their uncertainties as well as the total surface brightnesses were adopted from the SDSS DR1 archive. The Petrosian magnitudes and the total surface brightnesses are 

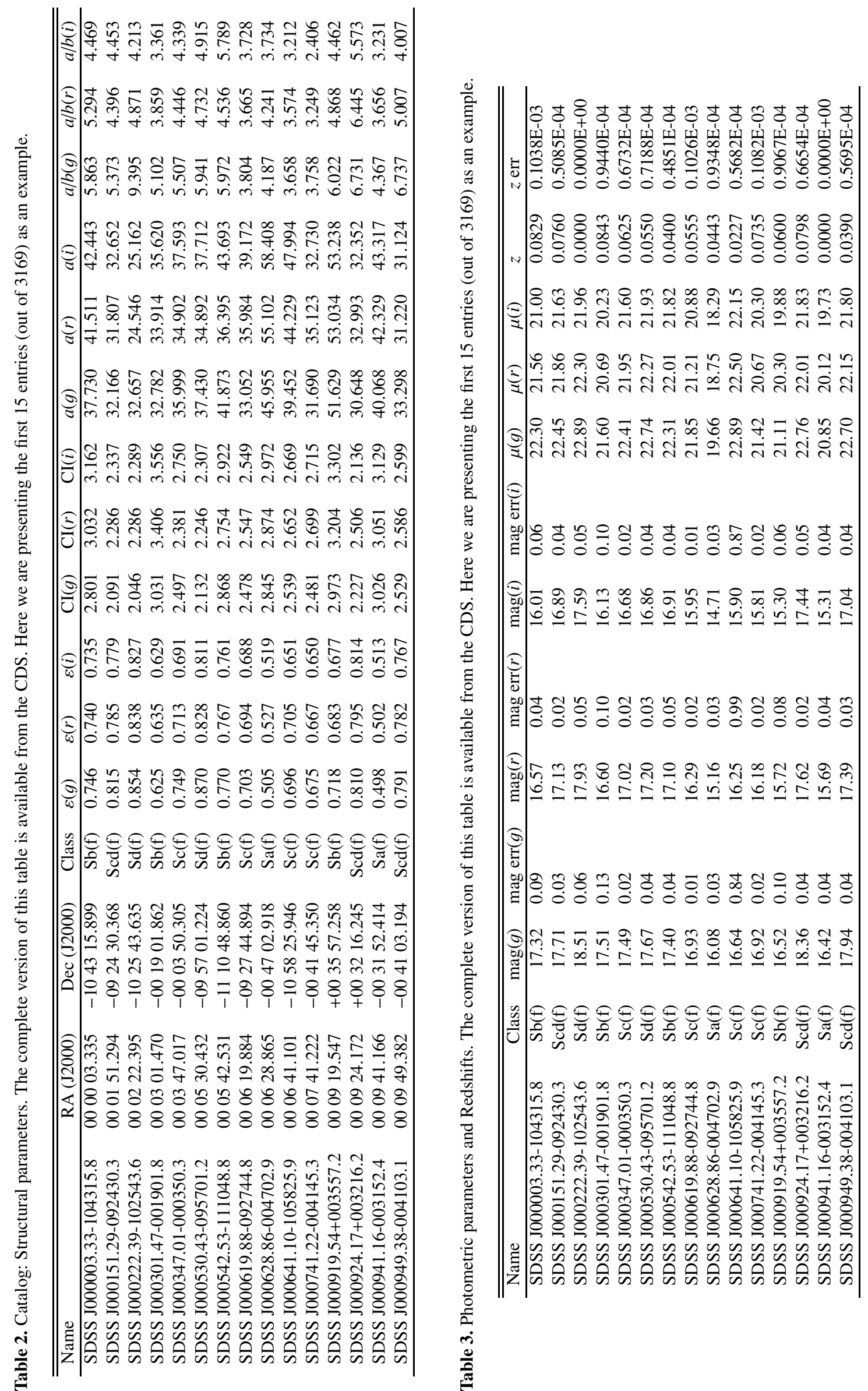


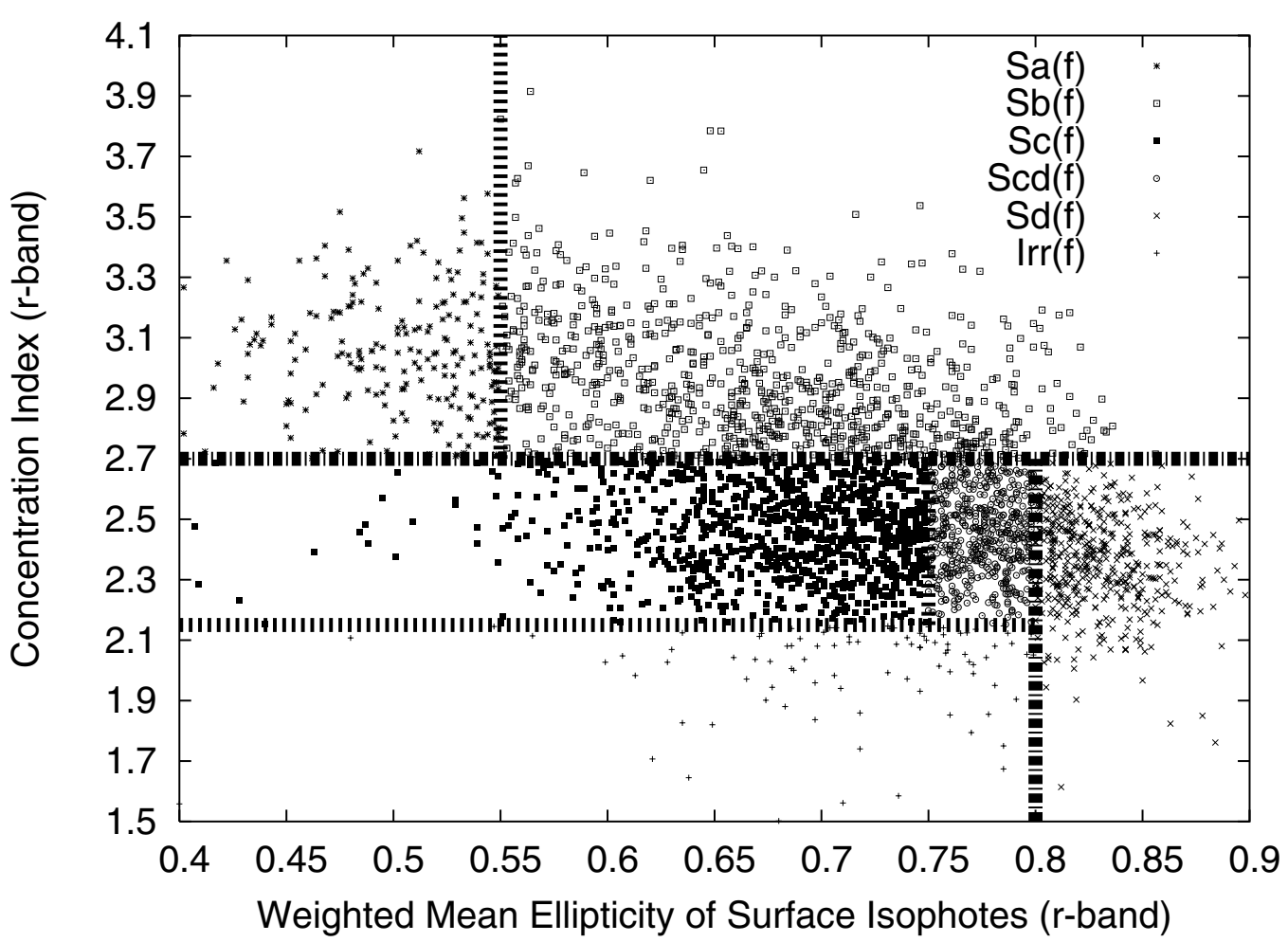

Fig. 4. The main separation diagram. The symbols are cited in the key and represents the various morphological types. The borders of the general classes are marked with long-short dotted lines. The additional short dotted lines corresponds to the subgroup borders within the general classes.

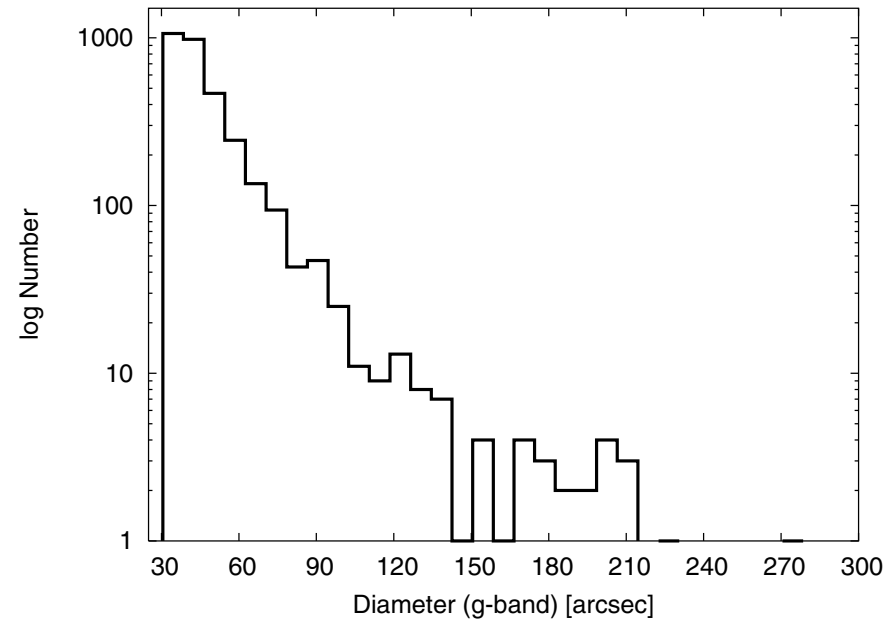

Fig. 5. Logarithmic number distribution of the angular diameters of the catalog galaxies.

corrected for Galactic foreground extinction with the extinction values of Schlegel et al. (1998) as given in the SDSS database.

If a value of the spectroscopic redshift $z$ and its uncertainty are available from the SDSS database, this measurement is listed in Cols. (12) and (13); otherwise these values are denoted as zero.

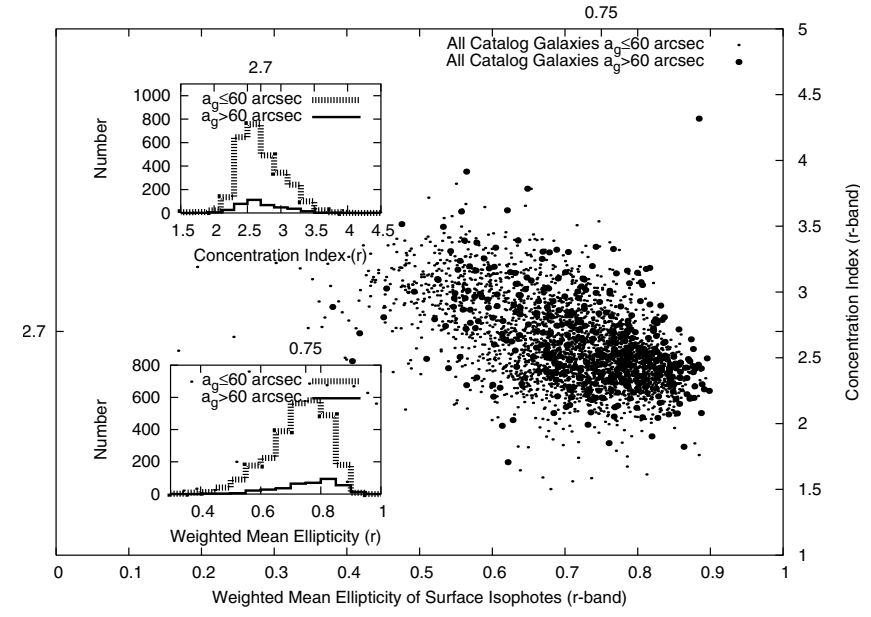

Fig. 6. Separation diagram with the emphasized size samples. Galaxies with angular diameters larger than 60 arcsec are indicated with filled points, smaller galaxies with fine dots. The upper inset shows the number distribution of the concentration index, the bottom inset that of the weighted mean ellipticity. Objects with a diameter $a \leqslant 60$ arcsec are denoted by the dashed line. The others are indicated by a filled black line.

\section{Completeness considerations from sample comparisons}

\subsection{A comparison of our automatically selected galaxy sample with our visually classified sample}

In order to estimate the completeness of our general morphological groups, we compare the results from our code with 
Table 4. Galaxy classes and their fractions. The absolute numbers of galaxies in the various morphological subclasses and their percentages with respect to the catalog entries as a whole are listed in this table.

\begin{tabular}{rrr}
\hline \hline General Class & Number & Percentages \\
\hline $\mathrm{Sa}(\mathrm{f})$ & 222 & 7.01 \\
$\mathrm{Sb}(\mathrm{f})$ & 843 & 26.60 \\
$\mathrm{Sc}(\mathrm{f})$ & 1005 & 31.71 \\
$\mathrm{Scd}(\mathrm{f})$ & 503 & 15.87 \\
$\mathrm{Sd}(\mathrm{f})$ & 501 & 15.81 \\
$\mathrm{Irr}(\mathrm{f})$ & 95 & 3.00 \\
Total & 3169 & 100.00 \\
\hline
\end{tabular}

those from the visual inspection using the reference set that we compiled to automate the separation process. Here we compare the three general classes, which are galaxies with bulge, intermediate types, and simple disk objects. According to the previously defined limiting values for the three general types, class $\mathrm{Sd}(\mathrm{f})$ contains 501 simple disks. The comparison with our visual galaxy classification shows an agreement of $97 \%$. Using our automated procedure, we identified 1065 objects from the bulge class ( $\mathrm{Sa}(\mathrm{f})$ and $\mathrm{Sb}(\mathrm{f})) .88 \%$ of these were also identified as clear bulge galaxies in our visually selected reference set. The intermediate class contains the largest fraction of galaxies (1603 objects). This class is not homogeneous and contains $\operatorname{Sc}(f), \operatorname{Scd}(f)$ and $\operatorname{Irr}(\mathrm{f})$ types. The comparison with the reference set indicates a completeness of $69 \%$, i.e., $31 \%$ belong to other classes using visual inspection. $88 \%$ of these $31 \%$ seem to belong to the simple disk class when classified by eye. This suggests that the automated classified intermediate class contains a large number of $\mathrm{Sd}(\mathrm{f})$ types.

The division between the classes is necessarily somewhat arbitrary, and galaxies close to a boundary may in many cases also be considered members of the adjacent class. For instance, uncertainties can be introduced by variations in galaxy properties which are a form of "cosmic noise". We required our visual subdivision to be consistent with the earlier studies (Karachentsev et al. 1993, 1999).

The number of $\mathrm{Sd}(\mathrm{f})$ objects is relatively low. However, we intentionally chose fairly conservative separation criteria in order to minimize possible contamination of our thus selected simple disk sample. If we use a more generous lower limiting value of $\varepsilon \geqslant 0.75$ and include the Scd(f) objects as simple disks, the simple disk object class contains 1004 galaxies (extended simple disk sample of seemingly bulgeless types, Scd(f) and $\mathrm{Sd}(\mathrm{f})$ ). This corresponds to $32 \%$ of the total catalog and enlarges the simple disk class by a factor of two. With this selection, however, the contamination by other types is larger than with the more rigorously defined limits for simple disks. Table 4 contains the absolute numbers and percentages of the various classes in comparison to the entries in the catalog as a whole.

\subsection{A comparison of the revised flat galaxies catalog with our catalog}

We searched for the RFGC galaxies in the SDSS DR1 using the coordinates given in the RFGC and recovered 328 objects.
Then we checked how many of these galaxies are recovered in our catalog using our selection criteria. We found 273 objects in common.

The remaining 55 RFGC galaxies were studied to find out why they were not recovered. In most cases objects are not recovered because they are not detected as SDSS targets ("Photoobjects"). This is the case when a galaxy is located near the borders of an SDSS stripe, which has the consequence that this object is not included in the "Best Galaxy Table" and subsequently not detected in the SDSS "Galaxy" catalog. In the cases of relatively extended objects these systems are "shredded" by the SDSS detection software and thus not included in our catalog. Furthermore, there are a few cases where the SDSS shows a galaxy with an inclination deviating from an edge-on orientation at a given set of RFGC coordinates. A small subset of RFGC galaxies are not really edge-on galaxies. If RFGC galaxies are very close to nearby bright foreground stars, they are also rejected by the SDSS software. We conclude that we recovered all RFGC galaxies that conform to our selection criteria except for those missed by the SDSS software and for those that are not edge-on. Hence the RFGC is more complete than our catalog for nearby (and hence seemingly large) edgeon systems. The RFGC is also more complete in terms of spatial coverage since it does not suffer from the detection problems near the bright stars or edges of stripes.

We plot the location of the recovered RFGC galaxies in our separation diagram in Fig. 7. It is clearly seen that most of the RFGC objects belong to the Scd(f) and Sd(f) class (184 of 273). Additionally, a smaller number of RFGC systems is found in the $\mathrm{Sb}(\mathrm{f}), \mathrm{Sc}(\mathrm{f})$ and $\operatorname{Irr}(\mathrm{f})$ classes. This is illustrated in the two inserted histograms in Fig. 7: RFGC objects clearly exceed our chosen limits for simple disk galaxies in the case of both discriminators, the CI (upper inset) and the $\varepsilon$ (bottom inset). That means that Karachentsev et al. (1993) and Karachentsev et al. (1999) did not only select simple disk systems, but also included some mixed-morphology galaxies, whose classification we can now correct thanks to the CCD data ${ }^{3}$.

With our separation routine we have thus improved the identification of simple disks in contrast to the flat galaxies catalogs. There are several reasons for this improvement: one is that the CCD images of the SDSS have a greater uniformity than the earlier employed blue POSS-I and ESO/SERC photo plates. In addition, the SDSS imaging data are deeper - they are $50 \%$ complete for point sources at $g=23.2 \mathrm{mag}$ (Abazajian et al. 2003). The limiting magnitude of the blue POSS-I plates is $20 \mathrm{mag}(\mathrm{R})$ (see Minkowski \& Abell 1963, p. 481), and of the $\mathrm{ESO} / \mathrm{SERC} \mathrm{J}$ plates is $22.5 \mathrm{mag}(\mathrm{B})$ (Reid et al. 1991). Because of the higher sensitivity, depth, and resolution of the SDSS, we can identify more substructure within our galaxies, which leads to a more accurate classification. This improved classification benefits from our choice of the SDSS $r$-band, which has the highest photon efficiency in the SDSS.

\footnotetext{
${ }^{3}$ Note, however, that our catalog contains all edge-on disk galaxies that we identified with our selection criteria, including mixedmorphology and bulge-dominated galaxies. The galaxy type can be found in Tables 2 and 3 as explained earlier.
} 


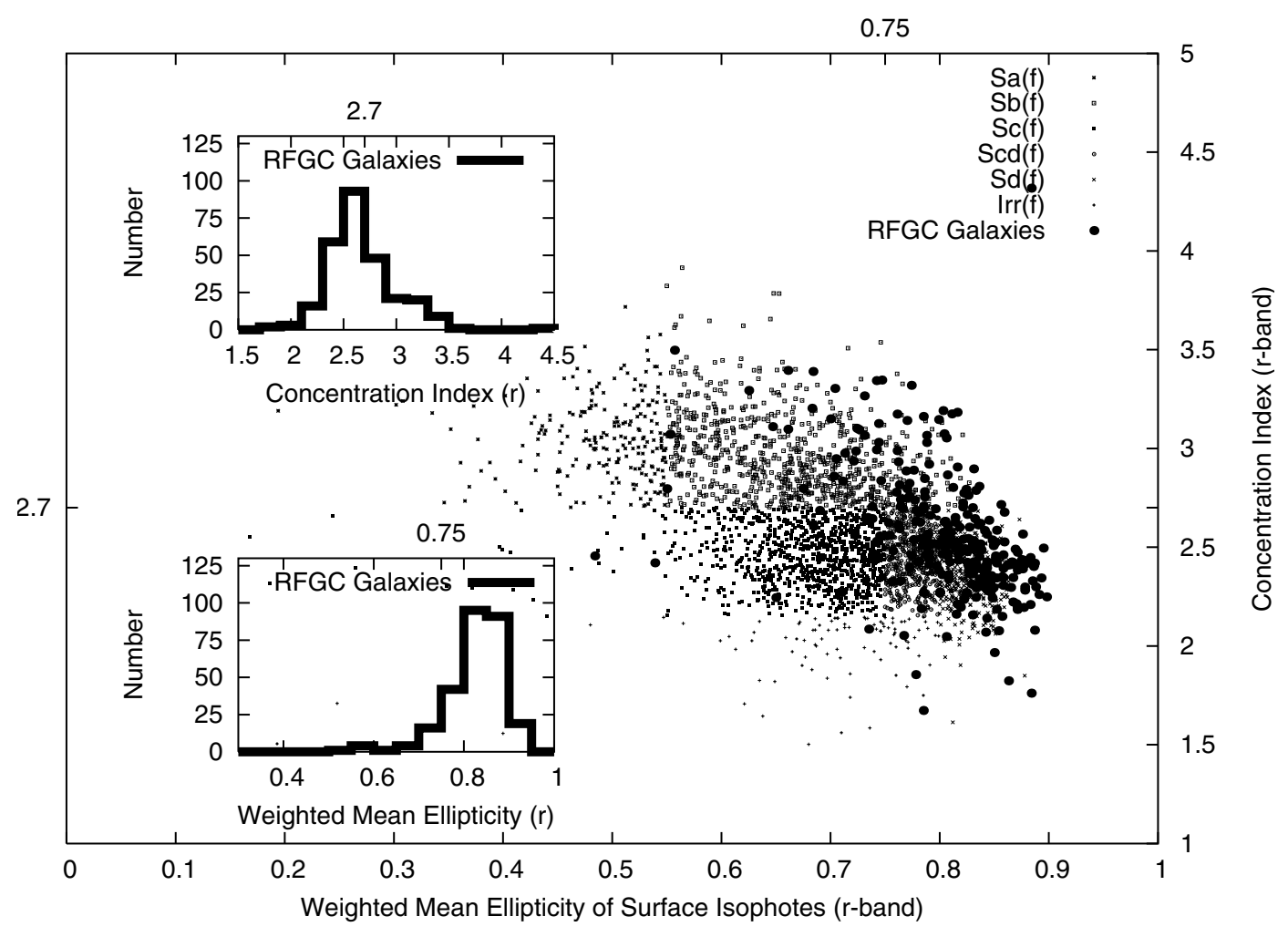

Fig. 7. Separation diagram with the recovered RFGC galaxies. Galaxies of the different morphological types are indicated by small symbols as given in the legend, recovered galaxies from the RFGC with large filled points. The upper inset shows the number distribution for the recovered RFGC galaxies of the concentration index, the bottom inset that of the weighted mean ellipticity.

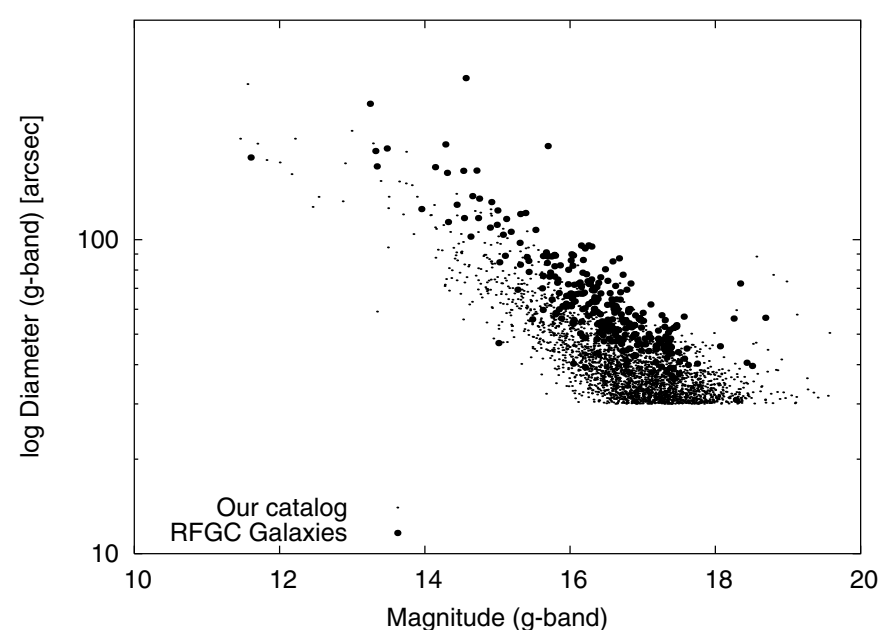

Fig. 8. Comparison of the depth of this catalog versus the recovered RFGC objects. The objects of the RFGC are large filled points, the catalog galaxies are small dots.

Our selection parameters include galaxies with smaller diameters and extend to fainter magnitudes. A comparison of the magnitudes and diameters of the recovered RFGC objects and the remaining galaxies from our catalog is shown in Fig. 8. As is to be expected, the figure shows that our catalog contains objects with smaller diameter as well as fainter objects. Furthermore, our catalog contains galaxies not detected in the RFGC but with diameters and luminosities in the range of the RFGC galaxies. Note that we also have a larger number of more

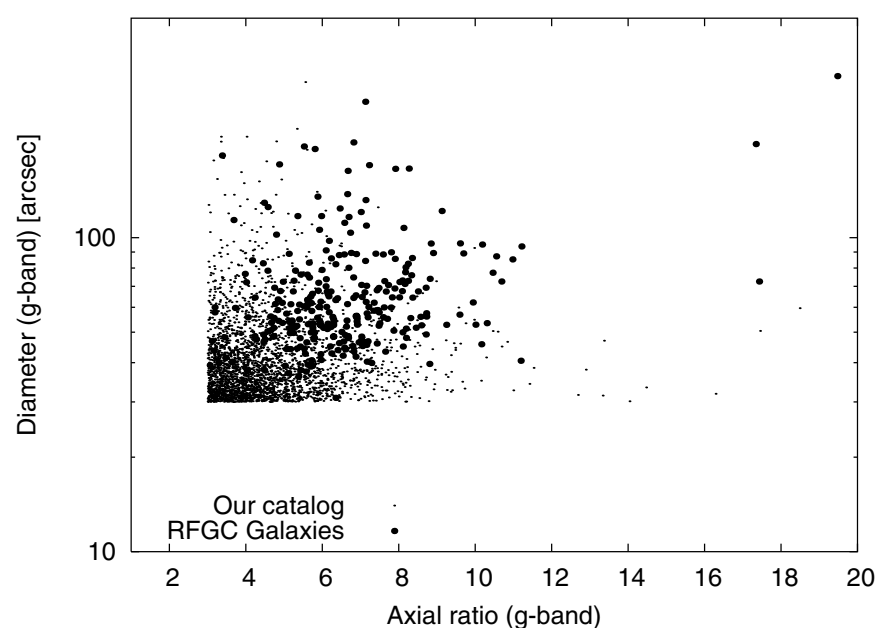

Fig. 9. Comparison of the depth of this catalog versus the recovered RFGC objects. The objects of the RFGC are large filled points, the catalog galaxies are small dots.

luminous galaxies at a given diameter. This is the consequence of permitting smaller axial ratios than the RFGC, which favors a larger number of disk galaxies with a bulge. Moreover, we show in Fig. 9, that in order to recover the RFGC galaxies from the SDSS database, we need to use selection criteria with smaller axis ratios. The SDSS axis ratios tend to be smaller than those of the RFGC. When comparing the number of seemingly bulgeless types (our $\operatorname{Scd}(\mathrm{f})$ and $\operatorname{Sd}(\mathrm{f})$ class with $\varepsilon \geqslant 0.75$ and $\mathrm{CI}<2.7$ ), our catalog contains 
1004 objects including 184 RFGC galaxies (out of 273 RFGC galaxies within the DR1 area). While we are missing galaxies near the edges of stripes etc., we still have $\sim 3.7$ times more simple disk galaxies than were found in the RFGC within the same area. As Fig. 8 illustrates, this is only in part because of the inclusion of smaller axis ratio. We attribute it also to the higher sensitivity and homogeneity of the SDSS.

It is difficult to determine absolute completeness numbers for our survey. For instance, in order to recover the initial RFGC training set, we had to decrease the angular diameters as compared to the parameters chosen in the RFGC. Furthermore, some incompleteness effects will affect all galaxies alike (e.g., the non-detection due to the location close to the border of a stripe), whereas for instance dust will affect certain galaxy types in particular (see next section).

\section{The influence of dust and distance}

We have subdivided our edge-on galaxies in objects with bulge and in simple disk systems without a bulge component. In this section we discuss the expected influence of dust on our separation procedure and a resolution bias caused by distance.

The distribution of dust in spiral galaxies has been the subject of a lively debate over decades. Recent studies try to model the influence of dust on the surface photometry. Kuchinsky et al. (1998) compared the optical/near infrared (NIR) color gradients of edge-on galaxies with the reddening from radiative transfer models. These models use Monte-Carlo techniques in order to describe the radiative transfer of photons (including scattering, absorption, and re-emission) in different dust environments (Gordon et al. 2001). Other models were applied to edge-on galaxy examples (see e.g., Xilouris et al. 1997, 1998; Pohlen et al. 2000; Popescu et al. 2000; Misiriotis \& Bianchi 2002). The best models include a homogeneous and clumpy distribution of dust (Kuchinsky et al. 1998). Tuffs et al. (2004) computed the attenuation of stellar light at different inclinations, wavelengths, and opacities from the different geometrical components of a spiral galaxy. They found that the extinction strongly depends on the inclination. In the case of edge-on systems most of the attenuation by dust occurs in the thin disk component, which often includes a typical dust lane.

But the amount of dust in different edge-on spiral types is not constant. This was recently shown by Stevens et al. (2005) with new SCUBA observations. Their measurements show that the flat galaxy NGC 5907 (FGC 1875) contains a very high amount of neutral hydrogen but only small amount of total dust. A high ratio of the mass of the neutral hydrogen to the mass of cold dust implies a very low star formation efficiency. In addition, Matthews et al. (1999) and Matthews \& van Driel (2000) show the "lack of a quintessential dust lane" in the prototypical superthin galaxy UGC 7321. In another paper, Matthews \& Wood (2001) conclude that the dusty interstellar medium (ISM) in this type of objects has a clumpy and patchy distribution. They derived the observed properties of dust with the aid of a multiphase ISM model and found that " $\approx 50 \%$ of the dusty material in UGC 7321 is contained in a clumpy medium". The other half has a diffuse distribution. UGC 7321 is an LSB galaxy with a large axial ratio and no bulge component, a typical simple disk.

What are the differences in the properties of edge-on galaxies with an organized dust lane and those that exhibit a clumpy and diffuse dust distribution? Dalcanton et al. (2004) found a clear boundary between edge-ons with and without a dust lane. They conclude that the dust distribution is connected with the rotation velocity, i.e., galaxy mass. Organized dust lanes appear in high surface brightness objects with a relative rapid rotation velocity. In galaxies with rotation velocities below $V_{\mathrm{c}}=120 \mathrm{~km} \mathrm{~s}^{-1}$ the dust has not settled into a thin dust lane. The dust distribution of these simple disk galaxies with typically a low surface brightness is clumpy and diffuse out to large scale heights.

What is the effect of a clumpy or an organized dust distribution on our separation values? In galaxies with a small angular size the dust lanes are unresolved, especially in those with larger distances. For these galaxies it is not a simple exercise to verify the existence of an intrinsic dust lane. In some of our larger and brighter galaxies a dust lane is visible, in others not. The dust is concentrated in the thin disk component and consequently the light attenuation caused by the thin disk is the highest (Tuffs et al. 2004). The stellar disk disappears in the extreme cases where a large amount of dust extends beyond the whole disk. Therefore the consequences of the dust attenuation of the disk are expected to be stronger in more massive galaxies like NGC 891. In these objects the probability of a dominant bulge is high. They are more metal-rich and are expected to contain more dust (mass-metallicity relation, e.g., Dalcanton et al. 2004). In general, the dust dims the thin disk with respect to the bulge brightness. This circumstance introduces three additional biases for our catalog:

1. Early-type disk galaxies: our catalog has an incompleteness for galaxies with big bulges and attenuated underlying thin disks. In these cases the disk cannot easily be detected as a bright structure because its morphology is like a dark line in a bright bulge component. Therefore this catalog is likely to be incomplete for dusty early-type spirals, especially for the types SO(f) and Sa(f), where we may be missing the disks. This may lead to an overestimate of the number of simple disks as compared to pronounced bulge-disk systems.

2. Massive late-type spirals/massive simple disks: a bias is shown in the extreme cases where the thin disk of a highmass simple disk galaxy is almost completely obscured by dust. In this case the galaxies may have too small an axial ratio $a / b$ to be selected for our catalog. In the less extreme cases of high-mass simple disk galaxies the dust dims the thin disk. The influence of dust in a simple disk is that the value of $\varepsilon$ decreases because a dusty disk appears to be thicker. For that reason, the affected galaxies may exhibit an offset toward the intermediate class in the separation diagram (Fig. 4). The CI of such galaxies is not distorted because these objects have no distinct bulge.

3. Objects at large distances are affected by a resolution bias. The consequences are that an unresolved dustlane dims the light of the stellar disk compared to a dust-free disk. This 
does not affect $\varepsilon$; the disk looks simply smaller. This may lead to a slight increase of the CI for disks with a bulge. However, the separation of the general classes does not seem to be displaced by the presence or absence of unresolved dust lanes. The important effect is that a strongly dimmed disk looks smaller and may fail to pass the selection criterion. The catalog will be more incomplete for small angular size disk galaxies with unresolved dust lanes. To reduce this effect we impose a minimum diameter in our search.

A future paper is planned in order to explore the effects of dust using simulated galaxies with varying amounts of dust and inclinations.

\section{Discussion}

We identified edge-on disk galaxies in the SDSS DR1, which we subdivide in the following classes:

Disk galaxies with a bulge; $\mathbf{S a}(\mathbf{f}), \mathbf{S b}(\mathbf{f})(\mathrm{CI} \geqslant 2.7)$ : the fraction of these objects in the catalog is $34 \%$. This class contains spiral galaxies with a bulge that are not affected by the selection effect described in Sect. 7.

Intermediate class; $\operatorname{Sc}(\mathbf{f}), \operatorname{Scd}(\mathbf{f}), \operatorname{Irr}(\mathbf{f})(\mathrm{CI}<2.7$ and $\varepsilon<$ 0.8 ): these late-type galaxies show a central light concentration and often a bouffant disk but no obvious bulge. With a fraction of $50 \%$ these types represent the majority in this catalog. These galaxies may have an inclination slightly smaller than edge-on or may show pronounced warps. At $\mathrm{CI}<2.15$ and $\varepsilon<0.8$ the class of disky edgeon (dwarf) irregulars appears. They show an asymmetric "puffy" disk with small clumpy (not central) light concentrations comparable to those found by Parodi et al. (2002) or blue compact dwarfs (e.g., Sandage \& Binggeli 1984); see also Kniazev et al. (2004a).

Simple disk galaxies; $\mathbf{S d}(\mathbf{f})(\mathrm{CI}<2.7$ and $\varepsilon \geqslant 0.8)$ : these galaxies appear to be pure bulgeless disks. Using the conservative separation values, this class contains $16 \%$ of the catalog objects.

In order to check the usefulness of our separation we visually inspected galaxies in the extreme regions in our separation diagram and found the following subgroups:

Dusty disk-dominated galaxies $(\mathrm{CI} \gtrsim 2.6$ and $\varepsilon \gtrsim 0.75)$ : these types have flat extended disks and slight central light concentration. The majority of this type appears as extended disks with dust lanes, small bulges and very blue outer disks.

Complex bulge/disk systems $(\mathrm{CI} \gtrsim 3$ and $\varepsilon<0.7)$ : these types are mostly complex bulge-disk systems. The bulge of these systems becomes clearly visible. In some of these types a stellar disk extends out to large scale heights and forms a bright but diffuse envelope around the galaxy.

In addition to the visual inspection of all galaxies in the extreme regions in the $\varepsilon-\mathrm{CI}$ space we also checked galaxies located in the central regions of the selection boxes for every general type by eye. In agreement with our expectations we found simple disk systems at high $\varepsilon$ and low CI $(0.8<\varepsilon<0.85$ and $2.3<\mathrm{CI}<2.4$ ). Their appearance is blue and needle-like. Intermediate values of $\mathrm{CI}$ and $\varepsilon$ reveal the region where intermediate types are concentrated $(0.7<\varepsilon<0.75$ and $2.5<\mathrm{CI}<$ 2.6). This group is dominated by lenticular-shaped puffy disks and smooth central light concentration but no dominant bulge component. The center is slightly redder than the bluer outer parts in these systems. It seems that they often have extended faint LSB disks around the bright parts. We checked the central region of disk galaxies with dominant bulges $(0.65<\varepsilon<0.7$ and $3<\mathrm{CI}<3.1)$. These early-type spirals are visibly less blue than the other general types in this catalog, and galaxies with bulge are the less well-populated group.

The highest concentration of galaxies can be found in the transition zone between the intermediate types and the simple disks. This indicates the lack of clear-cut boundaries between different types; instead we are seeing a continuum.

Figure 10 shows a comparison of the general classes with surface brightness. As shown in this figure, the presence or absence of bulges has an influence on the overall surface brightness of a galaxy as one would expect. Simple disk galaxies have the lowest intrinsic surface brightnesses of all edge-on galaxies in this catalog. For this plot we use the surface brightness given in Col. 10 of Table 3. This surface brightness is derived as explained in Sect. 5. No dust correction is applied.

\section{Conclusions and summary}

We present for the first time a homogeneous and large dataset of uniformly selected edge-on disk galaxies. These common galaxy types are very important in order to understand the formation and evolution of disk galaxies. The galaxies are selected from the SDSS DR1 on the basis of photometric structural parameters indicative of extended stellar disks with large major to minor axis ratios.

With the aid of this method we gathered 3169 edge-on galaxies in an area of $2099 \mathrm{deg}^{2}$ and selected through an automated separation algorithm. Using visual inspection of the galaxies we realized that this catalog contains three general classes of crude morphological types: disk galaxies with bulge, thin bulgeless objects and intermediate types.

The separation is based on the central light concentration and the flatness of the galaxy images. The light concentration is expressed via the concentration index CI. With this CI we are able to distinguish between disk galaxies with a bulge component $\mathrm{Sa}(\mathrm{f})$ and $\mathrm{Sb}(\mathrm{f})$ and those with bulgeless appearance - the $\operatorname{Sc}(f), \operatorname{Scd}(f), \operatorname{Sd}(f)$ and $\operatorname{Irr}(f)$ classes. As a second discriminator we use the luminosity-weighted average of the ellipticity $\varepsilon$, derived from elliptical isophotes of every object. The $\varepsilon$ allows one to distinguish several structural groups with flat disks: the early-type spirals, types $\mathrm{Sa}(\mathrm{f})$ from $\mathrm{Sb}(\mathrm{f})$; the apparently bulgeless systems from intermediate types $\operatorname{Sc}(f), \operatorname{Scd}(f)$ and $\operatorname{Irr}(f)$ with central light concentrations; and the thin, smooth galaxies, the simple disk class $\operatorname{Sd}(\mathrm{f})$.

The simple disk class includes objects previously defined as flat and superthin galaxies and it exhibits the lowest surface brightness compared to the other classes. The axial ratios of simple disk galaxies are the largest. The intermediate class of 


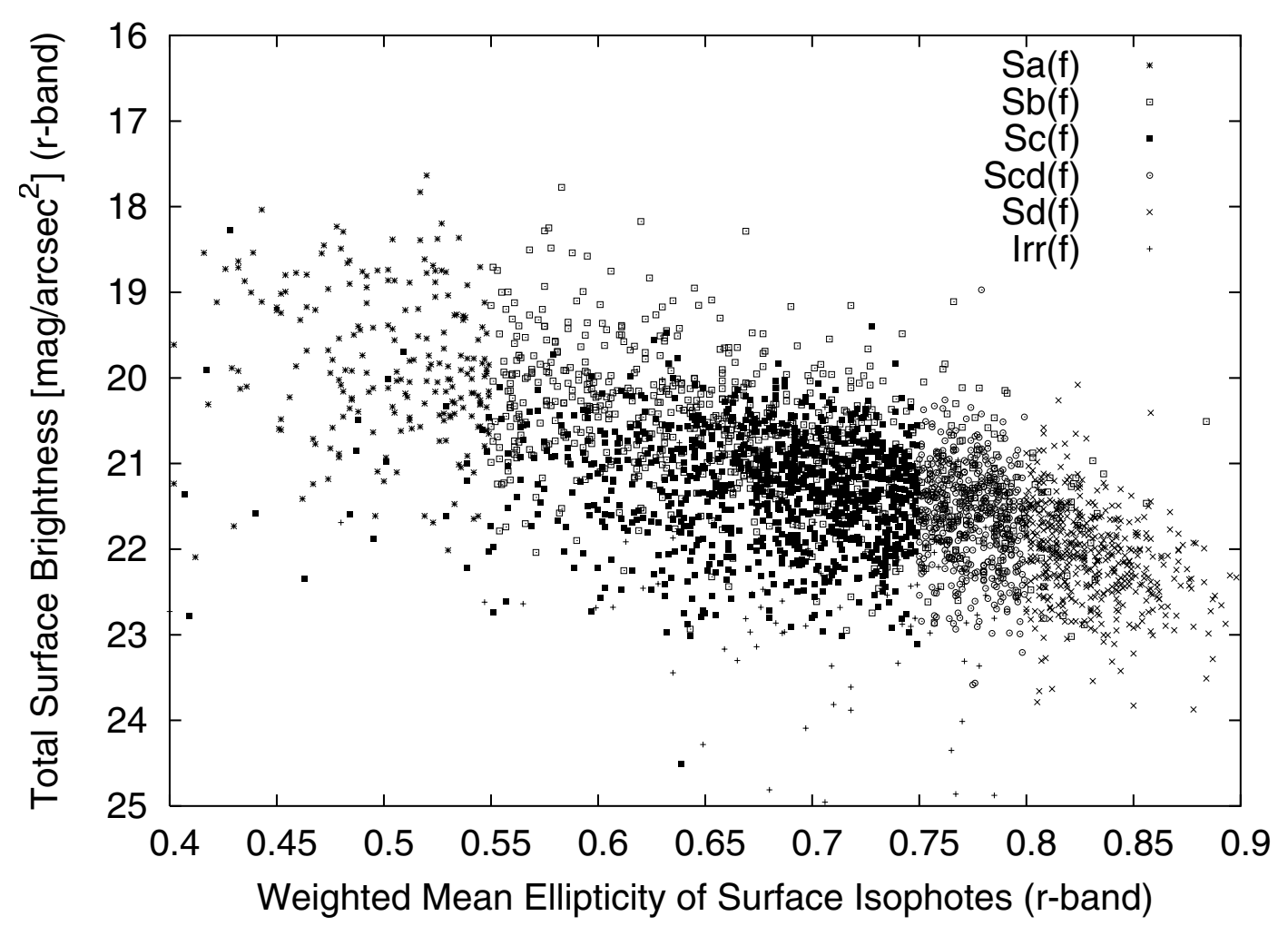

Fig. 10. Distribution of the $\varepsilon$ versus the total surface brightness. The different points represent the various morphological galaxy types and are explained in the key.

edge-ons is composed of different types of galaxies including (dwarf) irregular systems. There is no well-defined boundary between these general classes, but instead a continuum of properties exists.

The fraction of galaxies with bulge ( $\mathrm{Sa}(\mathrm{f}), \mathrm{Sb}(\mathrm{f}))$ is $34 \%$, those without bulge $16 \%(\operatorname{Sd}(\mathrm{f}))$ and the fraction of the intermediate class is $50 \%$. However, we found that the intermediate object class contains also a large fraction (about 440 objects, these are nearly $30 \%$ of the intermediates) of seemingly bulgeless types. Therefore we conclude that every general class (i.e., galaxies with bulge, intermediate objects and simple disks) represents about one third of the galaxies listed in the catalog. The true numbers of our classes are somewhat lower. Dust attenuation introduces a bias such that this catalog is expected to be incomplete for early-type spirals with pronounced dust lanes. Additional incompleteness is introduced because of features of the SDSS database such as galaxy shredding, etc.

In the case of late-type spirals, dust is expected in highmass systems and increases the apparent thickness of the disk. This results in a minor offset in the separation but has no effect on the completeness for simple disks. Unresolved dust lanes dim the disk light and lead to higher incompleteness for distant disk galaxies. A comparison with the RFGC shows that our catalog suffers from incompleteness for, e.g., galaxies close to bright stars or near the edge of a scan stripe, but that it nonetheless contains almost four times as many galaxies within a given area than the RFGC. This is mainly because we included also galaxies with smaller angular diameters, but it is also a result of the homogeneity, resolution, and depth of the SDSS.
This catalog provides a large, homogeneously selected galaxy sample for which sensitive five-color photometry (and in many cases also spectroscopy) is available. SDSS spectroscopy, while covering only a portion of the galaxies because of its circular aperture of $3^{\prime \prime}$, will be useful for a wide variety of studies, for instance for deriving metallicities and for constraining the properties of the underlying stellar populations (e.g., Kniazev et al. 2004b; Bernardi et al. 2005).

While these data will be analyzed in later papers, even the raw catalog data have interesting implications. Our results re-enforce the conclusions of Karachentsev and collaborators (Karachentsev et al. 1993, 1999) that simple disk galaxies are relatively common, especially among intermediate-mass starforming galaxies (Matthews \& Gallagher 1997). Galaxy formation models must be able to produce such high angular momentum systems with reasonable frequencies. We also find that the simple disks are not a separate morphological class, but rather are at the end of a continuum that extends smoothly from bulge+disk systems. However, the simple disks tend to be lower surface brightness galaxies, indicating that the probability for bulge formation depends on host galaxy mass. This in turn can be linked to models where bulges form from internal disk instabilities through the dependence of the Toomre Q-parameter on disk surface density (e.g., Immeli et al. 2004). Similarly the properties of our sample will be useful in constraining the role of galaxy mergers in building disk-halo galaxies (e.g., Springel \& Hernquist 2005; Kormendy \& Kennicutt 2004). We will explore these and related issues in future papers. 
Acknowledgements. S.J.K. and E.K.G. were supported by the Swiss National Science Foundation through the grants 200021-101924/1 and 200020-105260/1. JSG thanks the U.S. National Science Foundation for support through grant AST-9803018 to the University of Wisconsin, and the University's Graduate School for additional funding of this work. We thank our referee, Dr. Igor Karachentsev, for his thoughtful comments.

Funding for the creation and distribution of the SDSS Archive has been provided by the Alfred P. Sloan Foundation, the Participating Institutions, the National Aeronautics and Space Administration, the National Science Foundation, the U.S. Department of Energy, the Japanese Monbukagakusho, and the Max Planck Society. The SDSS Web site is http://www. sdss.org/.

The SDSS is managed by the Astrophysical Research Consortium (ARC) for the Participating Institutions. The Participating Institutions are The University of Chicago, Fermilab, the Institute for Advanced Study, the Japan Participation Group, The Johns Hopkins University, the Korean Scientist Group, Los Alamos National Laboratory, the Max-Planck Institute for Astronomy (MPIA), the Max-Planck Institute for Astrophysics (MPA), New Mexico State University, University of Pittsburgh, University of Portsmouth, Princeton University, the United States Naval Observatory, and the University of Washington.

\section{References}

Abadi, M. G., Navarro, J. F., Steinmetz, M., \& Eke, V. R. 2003, ApJ, 591,499

Abazajian, K., Adelman-McCarthy, J. K., Agüeros, M. A., et al. 2003, AJ, 126, 2081

Abazajian, K., Adelman-McCarthy, J. K., Agüeros, M. A., et al. 2004, AJ, 128, 502

Abazajian, K., Adelman-McCarthy, J. K., Agüeros, M. A., et al. 2005, AJ, 129, 1755

Abraham, R. G., van den Bergh, S., \& Nair, P. 2003, ApJ, 588, 218

Adelman-McCarthy, J. K., et al. 2006, ApJS, in press (January 2006 issue) [arXiv:astro-ph/0507711]

Athanassoula, E. 2005, MNRAS, 358, 1477

Bender, R., \& Moellenhof, C. 1987, A\&A, 177, 71

Bernardi, M., Sheth, R. K., Nichol, R. C., Schneider, D. P., \& Brinkmann, J. 2005, AJ, 129, 61

Blanton, M. R., Dalcanton, J., Eisenstein, D., et al. 2001, AJ, 121, 2358

Dalcanton, J. J., Spergel, D. N., \& Summers, F. J. 1997, ApJ, 482, 659

Dalcanton, J. J., Yoachim, P., \& Bernstein, R. A. 2004, ApJ, 608, 189

D’Onghia, E., \& Burkert, A. 2004, ApJ, 612, 13

Fall, S. M., \& Efstathiou, G. 1980, MNRAS, 193, 189

Gardner, J. P. 2001, ApJ, 557, 616

Goad, J. W., \& Roberts, M. S. 1979, BAAS, 11, 668

Goad, J. W., \& Roberts, M. S. 1981, ApJ, 250, 79

Gordon, K. D., Misselt, K. A., Witt, A. N., \& Clayton, G. C. 2001, ApJ, 551, 269

Immeli, A., Samland, M., Gerhard, O., \& Westera, P. 2004, A\&A, 413, 547

Karachentsev, I. 1989, AJ, 97, 1566

Karachentsev, I. D., Karachentseva, V. E., \& Parnovskij, S. L. 1993, Astron. Nachr., 314, 97

Karachentsev, I. D., Karachentseva, V. E., Kudrya, Yu. N., Sharina, M. E., \& Parnovsky, S. L. 1999, Bull. Special Astrophys. Obs., 47, 5

Kautsch, S. J., \& Grebel, E. K. 2003, Astron. Nachr. Suppl., 324 (S3), 149

Kelly, B. C., \& McKay, T. A. 2004, AJ, 127, 625
Kniazev, A. Y., Grebel, E. K., Pustilnik, S. A., et al. 2004a, AJ, 127, 704

Kniazev, A. Y., Pustilnik, S. A., Grebel, E. K., Lee, H., \& Pramskij, A. G. 2004b, ApJS, 153, 429

Kormendy, J., \& Kennicutt, R. C., Jr. 2004, ARA\&A, 42, 603

Kuchinsky, L. E., Terndrup, D. M., Gordon, K. D., \& Witt, A. D. 1998, AJ, 115, 1438

Kylafis, N. D., \& Xilouris, E. M. 2005, in The Spectral Energy Distributions of Gas-Rich Galaxies: Confronting Models with Data, ed. C. C. Popescu, \& R. J. Tuffs (Melville: AIP), AIP Conf. Proc., 761, 3

Lotz, J. M., Primack, J., \& Madau, P. 2004, AJ, 128, 163

Matthews, L. D., \& Gallagher, J. S. 1997, AJ, 114, 1899

Matthews, L. D., Gallagher, J. S., \& van Driel, W. 1999, AJ, 118, 2751

Matthews, L. D., \& van Driel, W. 2000, A\&AS, 143, 421

Matthews, L. D., \& Wood, K. 2001, ApJ, 548, 150

Matthews, L. D., \& de Grijs, R. 2004, AJ, 128, 137

Minkowski, R., \& Abell, G. O. 1963, Stars and Stellar Systems, Vol. 3, Basic Astronomical Data (Chicago: Univ. Chicago Press)

Misiriotis, A., \& Bianchi, S. 2002, A\&A, 384, 866

Mitronova, S. N., Karachentsev, I. D., Karachentseva, V. E., Jarrett, T. H., \& Kudrya, Yu. N. 2004, Bull. Special Astrophys. Obs., 57, 5

Nakamura, O., Fukugita, M., Yasuda, N., et al. 2003, AJ, 125, 1682

Noguchi, M. 2001, ApJ, 555, 289

Parodi, B. R., Barazza, F. D., \& Binggeli, B. 2002, A\&A, 388, 29

Petrosian, V. 1976, ApJ, 209, L1

Pohlen, M., Dettmar, R.-J., Lütticke, R., \& Schwarzkopf, U. 2000, A\&AS, 144, 405

Popescu, C. C., Misiriotis, A., Kylafis, N. D., Tuffs, R. J., \& Fischera, J. 2000, A\&A, 362, 138

Reid, I. N., Brewer, C., Brucato, R. J., et al. 1991, PASP, 103, 661

Reshetnikov, V. P. 1995, Astron. Astrophys. Trans., 8, 31

Robertson, B., Hernquist, L., Bullock, J. S., et al. 2005, preprint [arXiv: astro-ph/0503369]

Samland, M., \& Gerhard, O. E. 2003, A\&A, 399, 961

Samland, M. 2004, PASA, 21, 175

Sandage, A., \& Binggeli, B. 1984, AJ, 89, 919

Schlegel, D. J., Finkbeiner, D. P., \& Davis, M. 1998, ApJ, 500, 525

Schombert, J. M., Bothun, G. D., Schneider, S. E., \& McGaugh, S. S. 1992, AJ, 103, 1107

Shen, S., Mo, H. J., White, S. D. M., et al. 2003, MNRAS, 343, 978

Shimasaku, K., Fukugita, M., Doi, M., et al. 2001, AJ, 122, 1238

Sommer-Larsen, J., Goetz, M., \& Portinari, L. 2003, ApJ, 596, 47

Springel, V., \& Hernquist, L. 2005, ApJ, 622, L9

Steinmetz, M., \& Navarro, J. F. 2002, Nature, 7, 155

Steinmetz, M. 2003, Ap\&SS, 284, 325

Stevens, J. A., Amure, M., \& Gear, W. K. 2005, MNRAS, 357, 361

Stoughton, C., Lupton, R. H., Bernardi, M., et al. 2002, AJ, 123, 485

Strateva, I., Ivezic, Z., Knapp, G. R., et al. 2001, AJ, 122, 1861

Strauss, M. A., Weinberg, D. H., Lupton, R. H., et al. 2002, AJ, 124, 1810

Taylor, J. E., \& Babul, A. 2003, Ap\&SS, 284, 405

Tuffs, R. J., Popescu, C. C., Völk, H. J., Kylafis, N. D., \& Dopita, M. A. 2004, A\&A, 419, 821

de Vaucouleurs, G. 1959, Handbuch der Physik, Vol. 53, Astrophysik IV: Stellar Systems (Berlin, Goettingen, Heidelberg: SpringerVerlag)

Xilouris, E. M., Kylafis, N. D., Papamastorakis, J., Palealogou, E. V., \& Haerendel, G. 1997, A\&A, 325, 135

Xilouris, E. M., Alton, P. B., Davies, J. I., et al. 1998, A\&A, 331, 894 Yasuda, N., Fukugita, M., Narayanan, V. K., et al. 2001, AJ, 122, 1104 York, D. G., Adelman, J., Anderson, J. E., et al. 2000, AJ, 120, 1579 